\title{
Joint Next-Hop/Relay Selection for Distributive Multihop Cooperative Networks
}

\author{
Ahmed Alkhayyat \\ Department of Electronics and Communication Engineering, Cankaya University, 06530 Ankara, Turkey \\ Correspondence should be addressed to Ahmed Alkhayyat; al_kuptan@yahoo.com
}

Received 22 July 2015; Revised 14 October 2015; Accepted 15 October 2015

Academic Editor: Gabriella Bretti

Copyright (C) 2015 Ahmed Alkhayyat. This is an open access article distributed under the Creative Commons Attribution License, which permits unrestricted use, distribution, and reproduction in any medium, provided the original work is properly cited.

\begin{abstract}
Cooperative networks that use adjacent nodes to relay hearing data are employed as an effective technique to deal with channel fading, thereby enhancing the network performances. Next-hop and relay nodes selection schemes determine the optimal nexthop node toward the destination and relay node between the source and the optimal next-hop and/or destination. Offering joint next-hop and relay node selection in distributive multihop cooperative networks will enable the source to select the optimal nexthop node and relay node that can improve the system performance. In this paper, we propose a joint next-hop node and relay node selection (JNRS) protocol for wireless distributive multihop cooperative networks. The goal of JNRS is to reduce the spectral efficiency loss and outage probability. Analysis results show that JNRS can enhance the cooperative networks performance under general conditions compared to cooperative networks without the JNRS protocol.
\end{abstract}

\section{Introduction}

Distributive multihop cooperative networks have been considered in various areas to be promising networks for ubiquitous communication situations. In such networks, a sequence of multihop transmissions is required to transmit data from the source to the destination. As well, relay nodes that are intermediate nodes between source $(S)$ and destination $(D)$ can cooperate in each hop transmission using cooperative systems and can in turn provide spatial diversity gains. Diversity systems are well-known to offer an effective method of combating fading in wireless networks. Frequency, spatial, and time diversities are the three methods used in diversity systems [1]. It has been revealed that a scheme with multiple transmitter (input) and single receiver (output) antennas (MISO) enhance the received signal quality through diversity systems [2].

A different method to use separated antennas at the transmitter that can reach a similar spatial diversity gain is cooperative systems [3-5]. In cooperative systems, many nodes in a wireless network work together to form a virtual multiple antenna system. Adopting cooperation, it is potential to utilize the spatial diversity of the conventional MISO systems; however, it is not essential to have multiple antennas. The destination or next-hop $(\mathrm{NH})$ node receives multiple versions of the data from the source and relay nodes and combines these to obtain an additional reliable transmitted signal that can offer better performance. In distributive multihop cooperative networks, nodes can cooperate with each other to offer spatial diversity gain at the destination or the next-hop node. Here, any node can be a source, relay, next-hop, or destination. The purpose of a next-hop node is to help the source to reach the destination over multiplehop nodes even when the source and destination are not in the same range. As follows, the purpose of the relay node $(R)$ is to help in the transmission of the source data to the destination node. To guarantee diversity gains, the relay nodes are chosen in such way that their connection to the destination and/or next-hop node is independent of the connection to the source. In the context of cooperative systems, there are two main cooperative diversity systems for transmission between a couple of nodes over a multiple relay nodes, namely, the decode and forward (DF) [6] and amplify and forward (AF) protocols [7].

Although cooperative systems have some fundamental benefits compared to direct transmission mode (DTM), such 
as the diversity gain, the cooperative systems relaying the data and processing at the relay nodes produce some negative drawbacks. These include reduced spectral efficiency (spatial multiplexing gain) [8], increased delay during communication [9], and increased hardware complexity. Since retransmission of the data from the relay nodes to the next hop and/or destination reduces the spectral efficiency, researchers have focused on developing some techniques to alleviate the spectral efficiency loss. The use of space-time codes in a distributed manner in cooperatives systems was studied in [10]. Relay nodes perform full duplex communication; that is, relays can receive and transmit simultaneously [11]. Dynamic allocation of the time slots is studied in [12], and some best relay selection protocols are proposed in $[13,14]$. Practically, the implementation of distributed space code requires multiple antennas to be set up at the mobile set and this is not practical for small devices; full duplex cooperation requires the relay to cancel its self-interference from the received signal, but this is not robust in low cost radio devices. Dynamic allocation requires overhead and global information; relay selection is a simplistic way and does not require hard upgrading. It can achieve spectral efficiency and diversity gain.

An incremental redundancy protocol for $\mathrm{AF}$ and $\mathrm{DF}$ cooperative diversity was considered in [7], where the author proposed protocol that improves the spectral efficiency of wireless cooperative networks. The destination indicates success or failure by broadcasting a single bit of feedback to the source and relay nodes. If the source-destination signal-tonoise ratio is sufficiently high, the feedback indicates success of the direct transmission, and the relay node does nothing. If the source-destination signal-to-noise ratio is not sufficiently high for successful direct transmission, the feedback indicates unsuccessful of direct transmission and relay retransmits what was received from source using one of the transmission schemes AF or DF.

Relay node selection for DF cooperative diversity was considered in [15], where the author presented a relay node selection protocol based on link signal-to-noise ratio in wireless networks. The relay node decides when to retransmit based on signal-to-noise ratio between source-relay and relay-destination. Spectral efficiency loss reduction was not studied.

Relay node selection for DF cooperative diversity was considered in [16]. The author proposed relay node selection based on the harmonic mean of the links (channel coefficients of the links) between source-relay and relay-destination. In addition to relay node selection, the author considers incremental redundancy protocol that reduces the spectral efficiency loss.

Most previous work on distributive multihop cooperative networks scenarios $[7,15,16]$ (1) makes no try to select next-hop nodes, (2) selects next-hop nodes supposing that cooperating sets have been allocated a priori, and (3) uses relay node selection but does not try to accomplish the spectral efficiency loss reduction or next-hop node selection. There have been few studies on joint next-hop and relay nodes selection in distributive multihop cooperative networks.
We propose a selection protocol that chooses the maximum signal-to-noise ratio (SNR) links of the source-tonext hop $(S-\mathrm{NH})$, source-to-relay $(S-R)$, and relay-to-next hop $(R-\mathrm{NH})$; we refer to this as joint next-hop and relay nodes selection (JNRS) in distributive multihop cooperative networks and this system addresses the above problems. The JNRS protocol is summarized as follows: (1) the source determines the maximum SNR of $S$-NH link among the available links of next-hop nodes, (2) the next-hop node determines best relay node within the intermediate area, (3) the next-hop node compares the SNR of S-NH with the maximum SNRs of $S-R$ and/or $R-\mathrm{NH}$ links, and then (4) if maximum SNR links from $S-\mathrm{NH}$ are greater than maximum SNR of $S-R$ link and/or $R-N H$ links, the next-hop node transmits a positive acknowledgement (+ACK); otherwise, it transmits a negative acknowledgement ( $-\mathrm{ACK})$.

The rest of the paper is prepared as follows: in Section 2, we presented a detailed overview of the proposed JNRS protocol. The formulation and analysis of JNRS are provided in Section 3, while the spectral efficiency, the outage probabilities, and diversity order of the proposed JNRS protocol are evaluated, in Sections 3.1, 3.2, and 3.2.1, respectively. Performance and results are addressed in Section 4. Finally, Section 5 gives the conclusion and future work.

\section{Multihop Cooperative Structure Construction in JNRS}

2.1. Architecture Overview. In this subsection, the JNRS architecture is presented theoretically. JNRS protocol described below involves single $\mathrm{NH}$ and $R$ selection. Nodes in distributive multihop cooperative networks are distributed randomly over the service area; in our work, we assumed a uniform distribution to provide a convenient framework for analysis (see Figure 1). Let $G_{n}^{R_{m}}$ indicate the $m$ th relay nodes $\left(R_{m}\right)$ group in the $n$th stage and $G_{n}^{\mathrm{NH}_{k}}$ the $k$ th next-hop nodes $\left(\mathrm{NH}_{k}\right)$ group in the $n$th stage. In fact, the source and destination are separated by $n$th stages, where each stage consists of a relay nodes group $\left(G_{n}^{\mathrm{NH}_{k}}\right)$ and next-hop nodes group $\left(G_{n}^{R_{m}}\right)$. In contrast the last stage consists of a single hop to the destination, that is, only $G_{n}^{R_{m}}$ group.

We consider the DF protocol (i.e., decode and forward mode in cooperation without using JNRS), described as follows: in the first phase (first time slot) the source broadcasts the data to the receiver and relay node. In the second phase (second time slot), the relay node retransmits the data that was received from the source by the receiver. Receiver is combined with the received data via maximal ratio combining (MRC). In the sequel, the DF under the consideration of distributive multihop cooperative networks using JNRS (see Figure 2) is carried out as follows: in the first phase and at stage 1 , the source broadcasts the data to optimal $R_{1}$ and optimal $\mathrm{NH}_{2}$ (optimal relay node and nexthop node selection processes are given in Section 2.2). In the second phase, the optimal $R_{1}$ is chosen and it retransmits what is received from the source to the optimal $\mathrm{NH}_{2}$ and combines the received data via MRC. At stage 2 in the first phase, the optimal $\mathrm{NH}_{2}$ acts as the source and rebroadcasts 


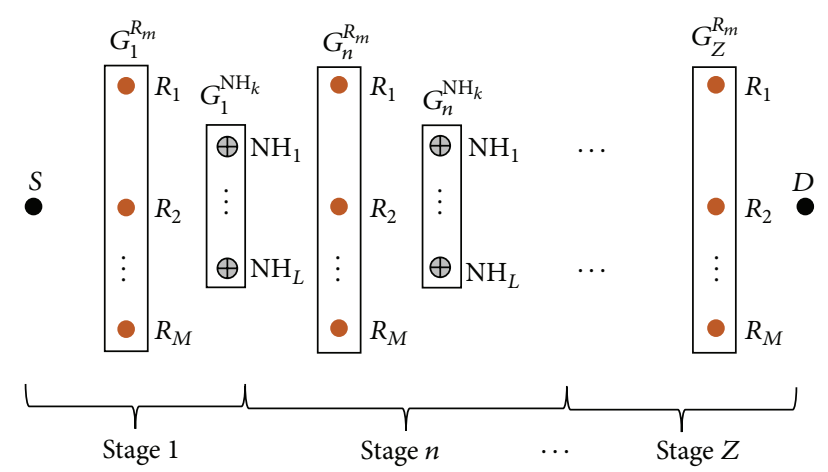

FIGURE 1: The distributive multihop cooperative networks scenario. There are $n$th stages between source and destination; each stage consists of the group of relay nodes $G_{n}^{R_{m}}$ and group of next-hop nodes $G_{n}^{\mathrm{NH}_{k}}$. Last stage $Z$ does not include a next-hop node group.

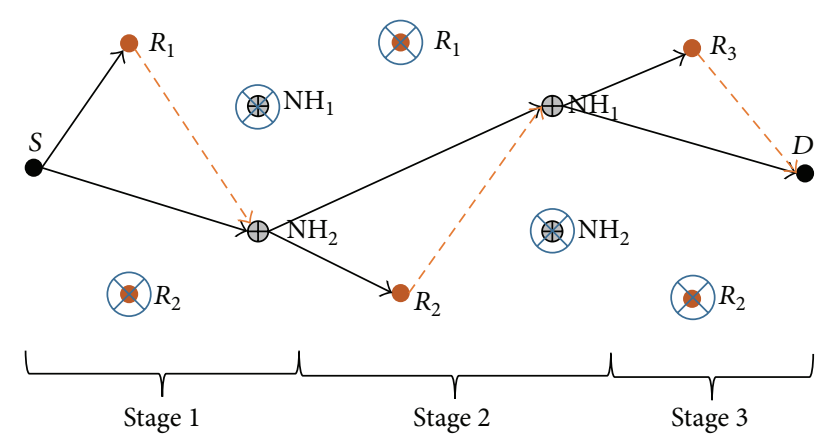

FIGURE 2: Example of distributive multihop cooperative networks, where the source and destination are separated by three stages $(Z=$ 3 ), and each stage consists of two relay nodes and two next-hop nodes. The sold and dashed lines represent the first phase and the second phase, respectively. The crossed nodes are not optimal and have been filtered out of communication.

the data received in stage 1 to the optimal $R_{2}$ and optimal $\mathrm{NH}_{1}$. In the second phase, the optimal $R_{2}$ retransmits what was received from the $\mathrm{NH}_{2}$ to the $\mathrm{NH}_{1}$, combines the received data via MRC, and so on. In stage 3, the next-hop node is the destination, where only the optimal relay node is selected. In this paper, we consider first stage in our analysis because it is assumed that the performance is identical at every stage because the last stage performance relies on previous stages (hops).

2.2. Next-Hop Node and Relay Node Selection. In distributive multihop cooperative networks, any source may have direct transmission links with some other nodes in its neighborhood and each node can, if needed, act as a next-hop node (router node) that routes the data to their final destination [17]. In addition, any transmitter may have intermediate nodes between itself and the receiver node, and these can function as relay nodes that help the transmitter to forward the data to the receiver (see Figures 1 and 2).

In this section, the JNRS is described in detail, with the a single next-hop node and single relay node jointly selected. Primarily, next-hop node selection is described (in fact, if the source and destination are within the same range, nexthop node does not exist). The NH node selection resembles the selection combing (SC) protocol in the diversity systems [18]. The SC is described as follows: the receiver received multiple signals from multiple transmitted antennas $(L)$ installed on the transmitter, where the receiver selects the largest SNR coming from $L$ transmitter antennas and filtered out the small SNRs. The proposed NH node selection is described as follows: the source determine multiple NH nodes within its neighborhood or within stage 1 in $G_{1}^{\mathrm{NH}_{k}}$ groups toward the destination; and because each of $S-\mathrm{NH}$ paths is independent sample of the fading process, the $S$-NH with the largest SNR is chosen for communication; this process can be expressed as follows:

$$
\gamma_{\max }^{S \mathrm{NH}} \begin{cases}1, & \gamma=\max \left\{\gamma_{k}\right\}, \\ 0, & \text { otherwize. }\end{cases}
$$

Here, $\gamma$ is the instantaneous SNR and $\gamma_{\max }^{S \mathrm{NH}}$ is the maximum instantaneous SNR of S-NH link. The NH node has been selected, and relay node selection is initiated. The proposed relay node selection is drawn from ARQ protocol, in which the destination transmits a negative ACK signal if the received data from the source are corrupted, making the source retransmits the corrupted data again. In contrast, if the data is received correctly by the destination, it transmits positive ACK signal. The proposed relay selection protocol is described as follows: in the first phase, the source broadcasts the data to optimal NH node which has been selected by the source and relay nodes (relay nodes within stage 1 in $G_{1}^{R_{m}}$ groups). In the second phase, the optimal NH node receives the source data, and it selects a best relay node with the largest SNR of $\gamma_{\max }^{S R, R \mathrm{NH}}$. This is expressed as follows:

$$
\begin{aligned}
\gamma_{\max }^{S R} & =\max \left\{\gamma^{S R_{1}}, \gamma^{S R_{2}}, \ldots, \gamma^{S R_{m}}\right\}, \\
\gamma_{\max }^{R N H} & =\max \left\{\gamma^{R N H_{1}}, \gamma^{R N H_{2}}, \ldots, \gamma^{R N H_{k}}\right\}, \\
\gamma_{\max }^{S R, R N H} & =\max \left\{\gamma_{\max }^{S R}, \gamma_{\max }^{R N H}\right\} .
\end{aligned}
$$

$\gamma_{\max }^{S R, R N H}$ represents the maximum SNR of the $S-R$ and $R-\mathrm{NH}$ links. If the $\gamma_{\max }^{S R, R N H}$ is determined, then the $\mathrm{NH}$ node compares it to $\gamma_{\max }^{S \mathrm{SH}}$. If $\gamma_{\max }^{S \mathrm{NH}}>\gamma_{\max }^{S R, R \mathrm{NH}}$, then the nexthop node transmits a positive ACK and DTM occurres; in contrast, if $\gamma_{\max }^{S \mathrm{NH}}<\gamma_{\max }^{S R, R \mathrm{NH}}$, then the NH node transmits negative ACK, and optimal relay node retransmits what was received from the source by the NH node (see Algorithm 1). The NH node is combined with the received data from the source and relay nodes using MRC.

We are interested in knowing the channel-state information (CSI), whether it is estimated or assumed to be known at each node. There is, of course, nothing wrong with making an optimistic assumption or perfect CSI (especially if it facilitates the analysis), as long as imposing this assumption does not change the core of the result from $[19,20]$. In this paper, it is assumed that the fading process is stationary; this is is called slow fading channel. The slow fading channel is constant for 


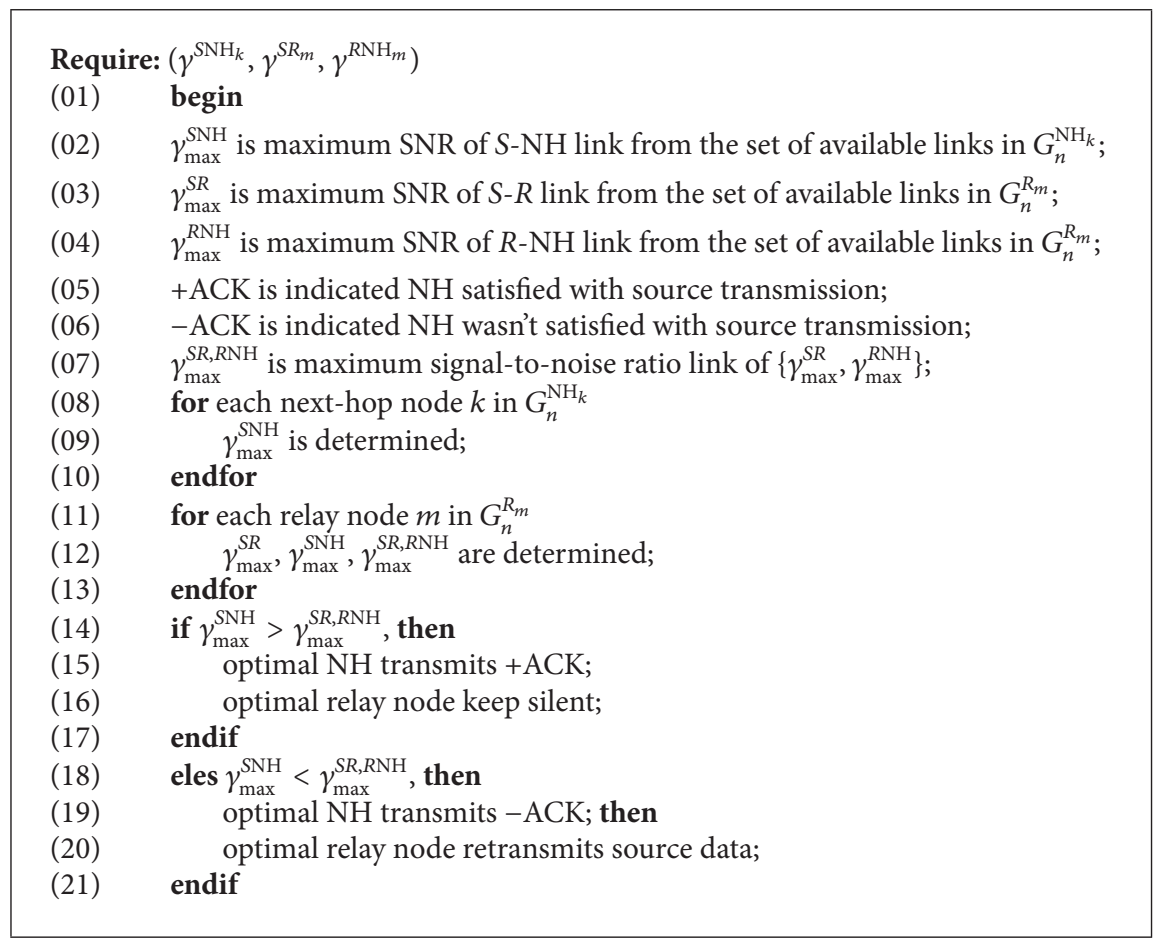

Algorithm 1: JNRS protocol description.

given symbol transmission (or fading varies slowly with time) and changes independently. Since the channel is varying slowly, the instantaneous CSI can be estimated, and this is feasible task. It is difficult to be aware of channel condition of $S-R$ and $R$-NH links of relay nodes at the NH node. But it will be easier if each relay node estimates the channel according to $S-R$ and $R-\mathrm{NH}$ conditions. To make channel condition estimation a feasible task, we use the scheme given in [21]. This selects the best relay between source and destination based on instantaneous channel measurements. The scheme given in [21] can be described briefly as follows: the relay nodes can overhear a signals transmission (hand shaking) of a ready-to-send (RTS) packet from $S$ and a clear-to-send (CTS) packet from $\mathrm{NH}$ for contention-based channel or using training sequences instead of RTS/CTS packets as hand shaking between $S$ and NH for control-based channel. After receiving the CTS packet, each relay node will start its own timer with an initial value $T_{m}$, which is inversely proportional to the end-to-end channel quality $h_{m}$; then, $T_{m}=1 / h_{m}$, in which $h_{m}=\max \left\{\gamma_{\max }^{S R}, \gamma_{\max }^{R N H}\right\}$. The first expired relay node transmits flag packet containing CSI of $S-R$ and $R-\mathrm{NH}$ to $\mathrm{NH}$ node. The $\mathrm{NH}$ node makes decision either to transmit $+\mathrm{ACK}$ or $-\mathrm{ACK}$ to the optimal relay node.

\section{Mathematical Analysis of the JNRS Protocol}

In this section, the mathematical model and analysis of JNRS protocol is provided. The average spectral efficiency, outage probability, and diversity order are analyzed after the JNRS protocol is employed.
3.1. Average Spectral Efficiency Analysis. Technically, if the $M$ relay nodes participate in cooperation, then $M+1$ time slots or subchannels are required to transmit a single symbol of the data from the source to the next-hop node and/or destination. Therefore, the spectral efficiency of the DF is scaled by $(1 / M+1)$; that is, if the spectral efficiency of DTM is denoted as $\mathrm{SE}_{\mathrm{DTM}}$, then the spectral efficiency of $\mathrm{DF}$ is $\mathrm{SE}_{\mathrm{DF}}=\left(\mathrm{SE}_{\mathrm{DTM}} /(M+1)\right)$.

After the optimal NH node is selected, the DF under JNRS may be summarized as follows: in the first phase, the source broadcasts the data to the relay nodes and optimal next-hop node; if the relay nodes decode the received data correctly, they save the data in their buffer and wait. Otherwise, the relay nodes keep silent and drop the data from the buffer. In second phase, the optimal $R$ transmits the flag packet to the optimal NH, which computes $\gamma_{\max }^{S N H}<\gamma_{\max }^{S R, R N H}$. If it is satisfied, the optimal next-hop transmits - ACK (the ACK message can be represented by single bit, where $+\mathrm{ACK}$ is by 1 digit and - ACK is by 0 digit, to avoid extra overhead). The optimal $R$ receives $-\mathrm{ACK}$ and it retransmits what was received from the source to optimal NH. The optimal NH combines all received signals that are transmitted by the source and the optimal $R$ via MRC. The JNRS can dramatically improve spectral efficiency over DF because if the source to optimal next hop node SNR is sufficiently high, the relay node does nothing. If the relay node does not participate in each direct transmission, the spectral efficiency loss can be reduced. The average spectral efficiency (ASE) can be expressed mathematically as follows [16]:

$$
\mathrm{ASE}=\underbrace{\operatorname{Pr}\left(\psi^{\mathrm{DTM}}\right)}_{\text {Term }(1)} \bigcup \underbrace{0.5 \operatorname{Pr}\left(\psi^{\mathrm{DF}}\right)}_{\text {Term }(2)} .
$$


The ASE after JNRS is employed is given as

$$
\begin{aligned}
& \mathrm{ASE}_{\mathrm{JNRS}} \\
& \quad=\underbrace{\operatorname{Pr}\left(\phi^{\mathrm{SNH}} \bigcap \psi^{\mathrm{DTM}}\right)}_{\text {Term }(3)} \bigcup \underbrace{0.5 \operatorname{Pr}\left(\phi^{\mathrm{SNH}} \bigcap \psi^{\mathrm{DF}}\right)}_{\operatorname{Term}(4)},
\end{aligned}
$$

in which $\phi^{\mathrm{SNH}}, \psi^{\mathrm{DTM}}$, and $\psi^{\mathrm{DF}}$ are optimal next-hop node selection event, direct transmission mode event which occurred at $\gamma_{\max }^{S \mathrm{NH}}>\gamma_{\max }^{S R, R N H}$, and DF mode event which occurred at $\gamma^{S \mathrm{NH}}<\gamma_{\max }^{S R, R \mathrm{NH}}$, respectively. Moreover, $\phi^{\mathrm{SNH}}$, $\psi^{\mathrm{DTM}}$, and $\psi^{\mathrm{DF}}$ are independent events. $\operatorname{Pr}(\cdot)$ is cumulative distribution function, where $\operatorname{Term}(3)$ and $\operatorname{Term}(4)$ are exclusively independents events. Therefore, we rewrite (4) as follows:

$$
\mathrm{ASE}_{\mathrm{JNRS}}=0.5\left[1+\operatorname{Pr}\left(\phi^{\mathrm{SNH}}\right) \operatorname{Pr}\left(\psi^{\mathrm{DTM}}\right)\right]
$$

in which $\operatorname{Pr}\left(\phi^{\mathrm{SNH}}\right)$ is the probability of optimal next-hop node selection and $\operatorname{Pr}\left(\psi^{\mathrm{DTM}}\right)$ is the probability of direct transmission mode selection; consequently, $\operatorname{Pr}\left(\psi^{\mathrm{DF}}\right)=1-$ $\operatorname{Pr}\left(\psi^{\mathrm{DTM}}\right)$ is probability of DF mode selection. If $\gamma$ is assumed to be exponentially distributed, then the complementary probability of optimal NH node selection is given as follows:

$$
\begin{aligned}
\overline{\operatorname{Pr}\left(\phi^{S N H}\right)} & =\overline{\operatorname{Pr}_{\gamma_{\max }}\left(\gamma^{S N H}\right)} \\
& =\operatorname{Pr}\left(\max \left[\gamma_{1}, \gamma_{2}, \ldots, \gamma_{L}\right] \geq \gamma^{S N H}\right) \\
& =\prod_{k=1}^{L}\left(1-\exp \left(-\frac{\gamma^{S N H}}{\gamma^{S N H}}\right)\right) ;
\end{aligned}
$$

this yields

$$
\overline{\operatorname{Pr}_{\gamma_{\max }}\left(\gamma^{S \mathrm{NH}}\right)}=\sum_{k=0}^{L}\left(\begin{array}{l}
L \\
k
\end{array}\right)(-1)^{k}\left(\exp -\frac{k \gamma^{S N H}}{\overline{\gamma^{S N H}}}\right) .
$$

The probability of optimal $\mathrm{NH}$ node selection is given as follows:

$$
\begin{aligned}
\operatorname{Pr}_{\gamma_{\max }}\left(\gamma^{S N H}\right)= & 1 \\
& -\sum_{k=0}^{L}\left(\begin{array}{l}
L \\
k
\end{array}\right)(-1)^{k}\left(\exp \left(-\frac{k \gamma^{S N H}}{\overline{\gamma^{S N H}}}\right)\right) .
\end{aligned}
$$

Furthermore, the probability of DTM selection is given as

$$
\begin{aligned}
\operatorname{Pr}\left(\psi^{\mathrm{DTM}}\right) & =\operatorname{Pr}_{\gamma_{\max }^{S R, R \mathrm{~N}}}\left(\gamma^{S \mathrm{NH}}>\gamma_{\max }^{S R, R N H}\right) \\
& =1-\exp \left(-\left(\frac{1}{\overline{\gamma^{S R}}}+\frac{1}{\overline{\gamma^{R N H}}}\right) \gamma^{S N H}\right) .
\end{aligned}
$$

However, if there is more than a single relay within $G_{1}^{R_{m}}$, the probability of DTM is given as

$$
\begin{aligned}
\operatorname{Pr}_{\gamma_{\max }^{S R, R N H}}\left(\gamma_{k}\right)= & \sum_{m=0}^{M}\left(\begin{array}{c}
M \\
m
\end{array}\right)(-1)^{m} \\
& \cdot \exp \left(-\left(\frac{1}{\overline{\gamma^{S R}}}+\frac{1}{\overline{\gamma^{R N H}}}\right) m \gamma^{S N H}\right) .
\end{aligned}
$$

The average $\operatorname{Pr}\left(\phi^{S N H}\right) \operatorname{Pr}\left(\psi^{\mathrm{DTM}} \mid \phi^{\mathrm{SNH}}\right)$ (i.e., for $\left.\gamma^{\mathrm{SNH}}>0\right)$ is given as

$$
\begin{aligned}
& \operatorname{Pr}\left(\phi^{S N H}\right) \operatorname{Pr}\left(\psi^{\mathrm{DTM}} \mid \phi^{S \mathrm{NH}}\right) \\
& =\int_{0}^{\infty} \operatorname{Pr}_{\gamma_{\max }}\left(\gamma^{S N H}\right) \operatorname{Pr}_{\gamma_{\max }^{S R, R N}}\left(\gamma_{k}\right) p_{\gamma^{S N H}}\left(\gamma^{S N H}\right) d \gamma^{S N H} .
\end{aligned}
$$

Substituting (8) and (10) in (11) yields

$$
\begin{gathered}
\operatorname{Pr}\left(\phi^{\mathrm{SNH}}\right) \operatorname{Pr}\left(\psi^{\mathrm{DTM}} \mid \phi^{S \mathrm{NH}}\right)=\int_{0}^{\infty} \sum_{m=0}^{M}\left(\begin{array}{l}
M \\
m
\end{array}\right)(-1)^{m} \\
\cdot \exp \left(-\left(\frac{1}{\overline{\gamma^{S R}}}+\frac{1}{\overline{\gamma^{R N H}}}\right) m \gamma^{S \mathrm{NH}}\right) \frac{1}{\overline{\gamma^{S N H}}} \\
\cdot \exp \left(-\frac{\gamma^{S \mathrm{NH}}}{\overline{\gamma^{S N H}}}\right) d \gamma^{S \mathrm{NH}}-\int_{0}^{\infty} \sum_{k=0}^{L}\left(\begin{array}{l}
L \\
k
\end{array}\right)(-1)^{k} \\
\cdot\left(\exp \left(-\frac{k \gamma^{S \mathrm{NH}}}{\overline{\gamma^{S N H}}}\right)\right) \underbrace{M}_{m=0}\left(\begin{array}{l}
M \\
m
\end{array}\right)(-1)^{m} \\
\cdot \exp \left(-\left(\frac{1}{\overline{\gamma^{S R}}}+\frac{1}{\overline{\gamma^{R N H}}}\right) m \gamma^{S N H}\right) \frac{1}{\overline{\gamma^{S N H}}} \\
\cdot \exp \left(-\frac{\gamma^{S \mathrm{NH}}}{\overline{\gamma^{S N H}}}\right) d \gamma^{S \mathrm{NH}}
\end{gathered}
$$

which then gives

$$
\begin{gathered}
\operatorname{Pr}\left(\phi^{S \mathrm{NH}}\right) \operatorname{Pr}\left(\psi^{\mathrm{DTM}} \mid \phi^{S \mathrm{NH}}\right)=\int_{0}^{\infty} \sum_{m=0}^{M}\left(\begin{array}{l}
M \\
m
\end{array}\right)(-1)^{m} \\
\cdot \exp \left(-\left(\frac{1}{\overline{\gamma^{S N H}}}+\left(\frac{1}{\overline{\gamma^{S R}}}+\frac{1}{\overline{\gamma^{R N H}}}\right) m\right) \gamma^{S \mathrm{NH}}\right) \\
\cdot \frac{1}{\overline{\overline{S N H}}} d \gamma^{S \mathrm{NH}}-\sum_{k=0}^{L}\left(\begin{array}{l}
L \\
k
\end{array}\right) \frac{(-1)^{k}}{\overline{\gamma^{S N H}}} \underbrace{M}_{m \neq k}\left(\begin{array}{l}
M \\
m
\end{array}\right)(-1)^{m} \\
\cdot \int_{0}^{\infty} \exp \left(-\left(\frac{(k+1)}{\overline{\gamma^{S N H}}}+\left(\frac{1}{\overline{\gamma^{S R}}}+\frac{1}{\overline{\gamma^{R N H}}}\right) m\right)\right. \\
\left.\cdot \gamma^{S \mathrm{NH}}\right) d \gamma^{S \mathrm{NH}} \cdot
\end{gathered}
$$

Evaluating the integration with respect to $\gamma^{\mathrm{SNH}}$, we rewrite (13) as

$$
\begin{gathered}
\operatorname{Pr}\left(\phi^{\mathrm{SNH}}\right) \operatorname{Pr}\left(\psi^{\mathrm{DTM}} \mid \phi^{\mathrm{SNH}}\right)=\frac{1}{\overline{\gamma^{S N H}}} \sum_{m=0}^{M}\left(\begin{array}{l}
M \\
m
\end{array}\right)(-1)^{m} \\
\cdot\left(\frac{1}{\overline{\gamma^{S N H}}}+\left(\frac{1}{\overline{\gamma^{S R}}}+\frac{1}{\overline{\gamma^{R N H}}}\right) m\right)^{-1} \\
-\sum_{k=0}^{L}\left(\begin{array}{l}
L \\
k
\end{array}\right) \frac{(-1)^{k}}{\overline{\gamma^{S N H}}} \underbrace{M}_{m \neq=0}\left(\begin{array}{l}
M \\
m
\end{array}\right)(-1)^{m} \\
\cdot\left(\frac{(k+1)}{\overline{\gamma^{S N H}}}+\left(\frac{1}{\overline{\gamma^{S R}}}+\frac{1}{\overline{\gamma^{R N H}}}\right) m\right)^{-1} .
\end{gathered}
$$


Substituting (14) in (4), the ASE of JNRS is given as follows:

$$
\begin{aligned}
\mathrm{ASE}_{\mathrm{JNRS}}=0.5\left(2+\frac{1}{\overline{\gamma^{S \mathrm{NH}}}} \sum_{m=1}^{M}\left(\begin{array}{l}
M \\
m
\end{array}\right)(-1)^{m}\right. \\
\cdot\left(\frac{1}{\overline{\gamma^{S \mathrm{NH}}}}+\left(\frac{1}{\overline{\gamma^{S R}}}+\frac{1}{\overline{\gamma^{R N H}}}\right) m\right)^{-1} \\
-\sum_{k=0}^{L}\left(\begin{array}{l}
L \\
k
\end{array}\right) \frac{(-1)^{k}}{\overline{\gamma^{S N H}}} \underbrace{M}_{m \neq k}\left(\begin{array}{c}
M \\
m
\end{array}\right)(-1)^{m} \\
. \\
\left.\quad\left(\frac{(k+1)}{\overline{\gamma^{S N H}}}+\left(\frac{1}{\overline{\gamma^{S R}}}+\frac{1}{\overline{\gamma^{R N H}}}\right) m\right)^{-1}\right) .
\end{aligned}
$$

It is clear that the ASE of the JNRS protocol depends on the following: (1) the number of the $\mathrm{NH}$ nodes within the $G_{1}^{\mathrm{NH}_{k}}$ group: if the number of $\mathrm{NH}$ nodes increases, the probability of choosing the optimal $\mathrm{NH}$ node increases, which reduces the spectral efficiency loss; in addition, the spectral efficiency approaches 1 as $L$ goes to $\infty$ and $M \neq$ $\infty$ because the probability (chance) of choosing maximum $S$-NH link approaches 1; (2) SNR of S-NH link: if the SNR of $S-\mathrm{NH}$ link is increased, then the probability of DTM increases as well, which reduces spectral efficiency loss; in addition, the spectral efficiency goes to 1 as $S$-NH goes to $\infty$; (3) the number of relay nodes within $G_{1}^{R_{m}}$ groups: the spectral efficiency decreases, if the number of relay nodes increases because as number of $R$ increases the probability of DF increases as well; in addition, the spectral efficiency goes to 0.5 as $M$ goes to $\infty$ and $L \neq \infty$ because the probability of existence maximum of SNR of $S-R$ and/or $R$-NH links is 1; (4) SNR of $S-R$ and $R-\mathrm{NH}$ links: the spectral efficiency decreases, if the SNR of $S-R$ and/or $R-\mathrm{NH}$ links is greater than $S-\mathrm{NH}$ link; in addition, the spectral efficiency goes to 0.5 as SNR of $S-R$ and/or $R-\mathrm{NH}$ links goes to $\infty$ since the probability of DF goes to 1 . We can conclude that if the probability of DTM increased, that is, $P\left(\psi^{\mathrm{DTM}}\right)$, the spectral efficiency increases, but the spectral efficiency reduces as $P\left(\psi^{\mathrm{DF}}\right)$ increases.

3.2. Outage Probability Analysis. The outage probability is another standard for system performance evaluation; it measures the probability of an event being less than the threshold value. In this section, we consider the probability of a given instantaneous SNR to be less than the threshold value $\gamma_{o}$. If the channel modeled as slow Raleigh fading channel, then the SNR distribution is exponential; therefore, the outage probability is given as follows [18]:

$$
\begin{aligned}
p\left(\gamma<\gamma_{o}\right) & :=\operatorname{Pr}\left(\gamma_{o}\right)=\int_{0}^{\gamma_{o}} \frac{1}{\bar{\gamma}} \exp \left(-\frac{\gamma}{\bar{\gamma}}\right) d \gamma \\
& =1-\exp \left(-\frac{\gamma_{o}}{\bar{\gamma}}\right)=\frac{1}{\bar{\gamma}} \exp \left(-\frac{\gamma_{o}}{\bar{\gamma}}\right),
\end{aligned}
$$

in which the $\bar{\gamma}$ is the average SNR and is given as $E[\gamma]=\beta \gamma$. The outage probability of the DF protocol is given as follows [21]:

$$
P_{\text {out }}^{\mathrm{DF}} \leq \underbrace{\left(P_{\text {out }}^{S \mathrm{NH}} \bigcap P_{\text {out }}^{S R}\right)}_{\text {First phase, Term }(5)} \bigcup \underbrace{\left(P_{\text {out }}^{S N H} \bigcap P_{\text {out }}^{R N H}\right)}_{\text {Second phase, Term }(6)} .
$$

The outage probability after JNRS is employed is given as follows:

$$
P_{\text {out }}^{\text {JNRS }} \leq \underbrace{\operatorname{Pr}\left(\phi^{S N H}\right) \bigcap P_{\text {out }}^{S R, S N H} \bigcap \operatorname{Pr}\left(\psi^{\mathrm{DTM}}\right)}_{\text {Term }(7)} \bigcup \underbrace{\operatorname{Pr}\left(\phi^{S N H}\right) \bigcap P_{\text {out }}^{R N H N H} \bigcap \operatorname{Pr}\left(\psi^{\mathrm{DF}}\right)}_{\text {Term }(8)} .
$$

All the events given in (18) are independents, and Term(7) and $\operatorname{Term}(8)$ are exclusively independent events. Therefore, we rewrite (18) as follows:

$$
\begin{aligned}
& P_{\text {out }}^{\text {JNRS }} \leq P_{\text {out }}^{S N H}\left[\operatorname{Pr}\left(\phi^{S N H}\right) \operatorname{Pr}\left(\psi^{\mathrm{DTM}}\right)\left(P_{\text {out }}^{S R}-P_{\text {out }}^{R N H}\right)\right. \\
& \left.\quad+\operatorname{Pr}\left(\phi^{S N H}\right) P_{\text {out }}^{R N H}\right],
\end{aligned}
$$

in which the average $\operatorname{Pr}\left(\phi^{\mathrm{SNH}}\right) \operatorname{Pr}\left(\psi^{\mathrm{DTM}}\right)$ is given in (14) and the average $\operatorname{Pr}\left(\phi^{\mathrm{SNH}}\right)$ is given as follows:

$$
\begin{aligned}
& P\left(\phi^{S \mathrm{NH}}\right)=1-\int_{0}^{\infty} \sum_{k=0}^{L}\left(\begin{array}{l}
L \\
k
\end{array}\right)(-1)^{k} \\
& \cdot \exp \left(-\frac{k \gamma^{S \mathrm{NH}}}{\overline{\gamma^{S N H}}}\right) \frac{1}{\overline{\gamma^{S N H}}} \exp \left(-\frac{\gamma^{S \mathrm{NH}}}{\overline{\gamma^{S N H}}}\right) d \gamma^{S \mathrm{NH}}=1 \\
& -\sum_{k=0}^{L}\left(\begin{array}{l}
L \\
k
\end{array}\right)(-1)^{k} \frac{1}{\overline{\gamma^{S N H}}}\left(\frac{\left.\frac{k+1}{\overline{\gamma^{S N H}}}\right)^{-1}}{}\right.
\end{aligned}
$$

in which $P_{\text {out }}^{S \mathrm{NH}}, P_{\text {out }}^{S R}$, and $P_{\text {out }}^{R \mathrm{NH}}$ are the outage probabilities of the $S$-NH links, $S-R$ link, and $R-\mathrm{NH}$ links, respectively. These probabilities are given as

$$
\begin{aligned}
P_{\text {out }}^{S N H} & =\operatorname{Pr}\left(\gamma^{S \mathrm{NH}}>\gamma_{o}\right)=1-\exp \left(-\frac{\gamma_{o}}{\overline{\gamma^{S \mathrm{NH}}}}\right) \\
& =g\left(\gamma^{S \mathrm{NH}}, \gamma_{o}\right), \\
P_{\text {out }}^{S R} & =\operatorname{Pr}\left(\gamma^{S R}>\gamma_{o}\right)=1-\exp \left(-\frac{\gamma_{o}}{\overline{\gamma^{S R}}}\right) \\
& =g\left(\gamma^{S R}, \gamma_{o}\right), \\
P_{\text {out }}^{R N H} & =\operatorname{Pr}\left(\gamma^{R N H}>\gamma_{o}\right)=1-\exp \left(-\frac{\gamma_{o}}{\overline{\gamma^{R N H}}}\right) \\
& =g\left(\gamma^{R \mathrm{NH}}, \gamma_{o}\right) .
\end{aligned}
$$


Substituting (14), (20), and (21) in (19), the outage probability obtain as follows:

$$
\begin{aligned}
& P_{\text {out }}^{\text {JNRS }} \leq g\left(\gamma^{\text {SNH }}, \gamma_{o}\right)\left[\frac{1}{\overline{\gamma^{S N H}}} \sum_{m=0}^{M}\left(\begin{array}{c}
M \\
m
\end{array}\right)(-1)^{m}\right. \\
& \cdot\left(\frac{1}{\overline{\gamma^{S N H}}}+\left(\frac{1}{\overline{\gamma^{S R}}}+\frac{1}{\overline{\gamma^{R N H}}}\right) m\right)^{-1} \\
& -\sum_{k=0}^{L}\left(\begin{array}{l}
L \\
k
\end{array}\right) \frac{(-1)^{k}}{\overline{\gamma^{S N H}}} \underbrace{\sum_{m=0}^{M}}_{m \neq k}\left(\begin{array}{l}
M \\
m
\end{array}\right)(-1)^{m} \\
& \cdot\left(\frac{(k+1)}{\overline{\gamma^{S \mathrm{NH}}}}+\left(\frac{1}{\overline{\gamma^{S R}}}+\frac{1}{\gamma^{R \mathrm{NH}}}\right) m\right)^{-1} \\
& \cdot\left(g\left(\gamma^{S R}, \gamma_{o}\right)-g\left(\gamma^{R N H}, \gamma_{o}\right)\right)+g\left(\gamma^{R N H}, \gamma_{o}\right) \\
& \left.\cdot\left(1-\sum_{k=0}^{L}\left(\begin{array}{l}
L \\
k
\end{array}\right)(-1)^{k} \frac{1}{\overline{\gamma^{S \mathrm{NH}}}}\left(\frac{k+1}{\overline{\gamma^{S \mathrm{NH}}}}\right)^{-1}\right)\right] .
\end{aligned}
$$

The outage probability is directly proportional to the probability of DTM and the probability of DF. This is affected by four parameters, which can be summarized as follows: (1) The outage probability reduces as $L$ increases because the probability of choosing the optimal $S$-NH link increases; otherwise, the outage probability increases. (2) The outage probability reduces as $S$-NH link increases, and vice versa. (3) The outage probability reduces as $M$ increases because the probability of choosing optimal $S-R$ and $R-\mathrm{NH}$ links increases; on other hand, the outage probability reduces as $M$ reduces. (4) The outage probability reduces as SNR $S-R$ and/or $R$-NH links increase and vice versa. (5) The outage probability reduces more compared to points $1-4$ if SNR of $S$-NH, $S-R$, and $R-\mathrm{NH}$ links all increases together.

3.2.1. Diversity Order. A good cooperation system has to maintain the diversity gain and spectral efficiency. In fact, DF may provide diversity gain but not spectral efficiency. Hence, we need a protocol that can provide both spectral efficiency and diversity gain. One of the simplest existing protocols that can reduce the spectral efficiency loss resembles ARQ protocol, but such protocol may not offer diversity gain. Therefore, it is important to design protocol that resembles ARQ protocol which offers diversity gain. The diversity order (DO) is considered in our analysis, because it can be translated into the diversity gain.

Definition 1. If the symbol of the data is transmitted from the source and optimal single relay node $(M=1)$ to the destination node (or next-hop node), one says that $\mathrm{DO}=2$, if $\gamma \rightarrow \infty$.

Proposition 2. The diversity order of JNRS that employs $M$ relay nodes achieves a diversity order of 2 through choosing maximum SNR from $S-N H, S-R$, and $R-N H$.
Proof. The probability density function of outage probability of the JNRS protocol is given as

$$
\begin{aligned}
& p_{\text {out }}^{\text {JNRS }} \\
& \leq p_{\text {out }}^{S N H} p\left(\phi^{S N H}\right)\left[p\left(\psi^{\mathrm{DTM}}\right)\left(p_{\text {out }}^{S R}-p_{\text {out }}^{R N H}\right)+p_{\text {out }}^{R N H}\right]
\end{aligned}
$$

in which $p_{\text {out }}^{S \mathrm{NH}}, p_{\text {out }}^{S R}$, and $p_{\text {out }}^{R \mathrm{NH}}$ are given as

$$
\begin{aligned}
p_{\text {out }}^{S \mathrm{NH}} & :=\operatorname{Pr}\left(\gamma^{S \mathrm{NH}}>\gamma_{o}\right)=1-\exp \left(-\frac{\gamma_{o}}{\overline{\gamma^{S \mathrm{NH}}}}\right) \\
& \sim \frac{\gamma_{o}}{\overline{\gamma^{S \mathrm{NH}}}}, \\
p_{\text {out }}^{S R} & :=\operatorname{Pr}\left(\gamma^{S R}>\gamma_{o}\right)=1-\exp \left(-\frac{\gamma_{o}}{\overline{\gamma^{S R}}}\right) \frac{\gamma_{o}}{\overline{\gamma^{S R}}}, \\
p_{\text {out }}^{R N H} & :=\operatorname{Pr}\left(\gamma^{R \mathrm{NH}}>\gamma_{o}\right)=1-\exp \left(-\frac{\gamma_{o}}{\overline{\gamma^{R N H}}}\right) \\
& \sim \frac{\gamma_{o}}{\overline{\gamma^{R N H}}} .
\end{aligned}
$$

The probability density functions of $\operatorname{Pr}\left(\phi^{\mathrm{SNH}}\right)$ and $\operatorname{Pr}\left(\psi^{\mathrm{DTM}}\right)$ for $L=1$ and $M=1$ are given as

$$
\begin{aligned}
& p_{\gamma_{\max }}\left(\gamma^{S \mathrm{NH}}\right):=\operatorname{Pr}_{\gamma_{\max }}\left(\gamma^{S \mathrm{NH}}\right) \\
& =1-\sum_{k=0}^{L}\left(\begin{array}{l}
L \\
k
\end{array}\right)(-1)^{k}\left(\exp \left(-\frac{k \gamma^{S \mathrm{NH}}}{\overline{\gamma^{S N H}}}\right)\right) \sim \frac{\gamma^{S \mathrm{NH}}}{\overline{\gamma^{S N H}}}, \\
& p_{\gamma_{\max }^{S R, R \mathrm{NH}}}\left(\gamma_{k}\right):=\operatorname{Pr}_{\gamma_{\max }^{S R, R \mathrm{NH}}}\left(\gamma_{k}\right) \\
& =\sum_{m=0}^{M}\left(\begin{array}{l}
M \\
m
\end{array}\right)(-1)^{m} \exp \left(-\left(\frac{1}{\overline{\gamma^{S R}}}+\frac{1}{\overline{\gamma^{R N H}}}\right) m \gamma^{S \mathrm{NH}}\right) \\
& \sim \gamma^{S \mathrm{NH}}\left(\frac{1}{\overline{\gamma^{S R}}}+\frac{1}{\overline{\gamma^{R N H}}}\right) .
\end{aligned}
$$

Substituting (24) and (25) in (23), obtain

$$
\begin{aligned}
& p_{\text {out }}^{\mathrm{JNRS}} \leq\left(\frac{k \gamma_{o}^{2}}{\gamma \beta^{\mathrm{SNH}}}\right) \\
& \cdot\left[\left(\left(\frac{1}{\beta^{S R}}+\frac{1}{\beta^{R N H}}\right) m\right)\left(\frac{1}{\gamma \beta^{S R}}-\frac{1}{\gamma \beta^{R N H}}\right)\right. \\
&\left.+\frac{1}{\gamma \beta^{R N H}}\right]
\end{aligned}
$$

in which $\beta^{S \mathrm{NH}}, \beta^{S R}$, and $\beta^{R N H}$ are the average of the squared of Raleigh random variable of the $S-\mathrm{NH}, S-R$, and $R-\mathrm{NH}$ links, respectively. In what follows, the diversity order of the JNRS protocol is given as follows:

$$
\mathrm{DO}_{\text {JNRS }}=-\lim _{\gamma \rightarrow \infty} \frac{\log \left(p_{\text {out }}^{\mathrm{JNRS}}\right)}{\log (\gamma)} .
$$

Substituting (26) in (27) and evaluating limit, the diversity order is equal to 


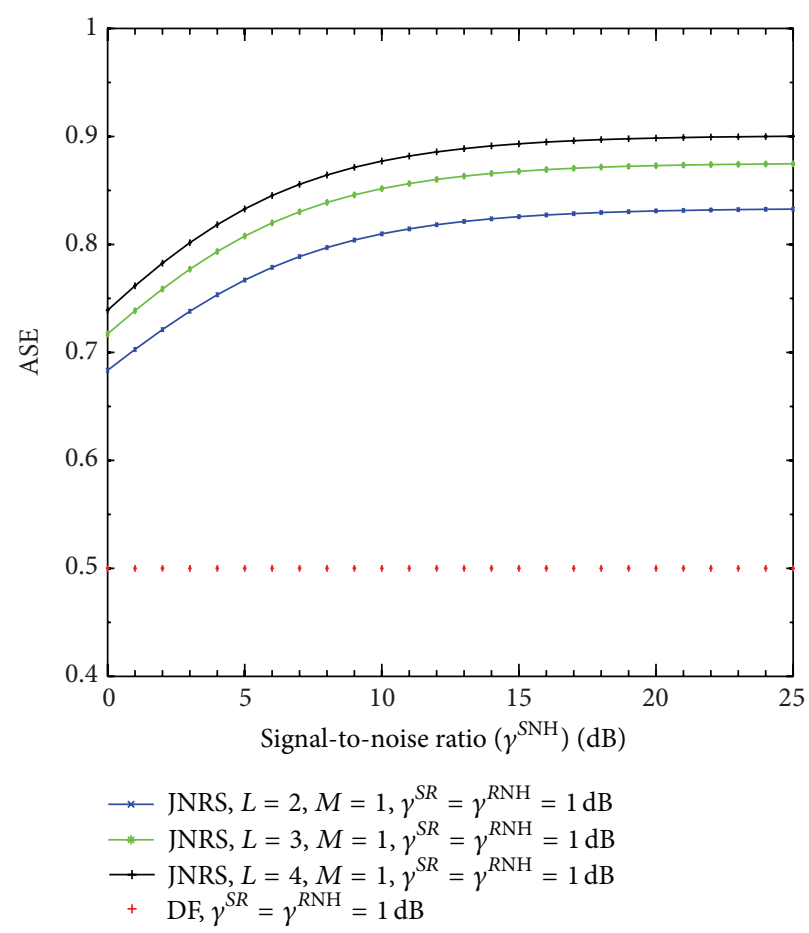

FIgURE 3: The ASE for $L=1,2$ and $M=1$ and different SNR.

$$
\mathrm{DO}^{\mathrm{INRS}}=-\lim _{\gamma \rightarrow \infty} \frac{\log \left(\left(k \gamma_{o}^{2} / \gamma \beta^{S \mathrm{NH}}\right)\left[\left(\left(1 / \beta^{S R}+1 / \beta^{R N H}\right) m\right)\left(1 / \gamma \beta^{S R}-1 / \gamma \beta^{R N H}\right)+1 / \gamma \beta^{R N H}\right]\right)}{\log (\gamma)}=2 .
$$

\section{Performance and Results}

In this section, the performance of the JNRS protocol presented in the previous sections is evaluated. The evaluations in this paper are gathered spectral efficiency and outage probability using JNRS protocol. In the evaluation, we assumed different SNR between the source, next-hop, and the relay nodes, which is the most general case. However, in Figure 3, we assume the SNR in S-NH is varying and between $S-R$ and $R-\mathrm{NH}$ is fixed, while in Figure 4 the SNR in $S-\mathrm{NH}$ is fixed and between $S-R$ and $R-\mathrm{NH}$ links is varying. In Figure 5, the $\mathrm{SNR}$ between $S-\mathrm{NH}, S-R$, and $R-\mathrm{NH}$ all varies.

Figure 3 show the comparison of ASE using JNRS protocol and DF for $L=2,3$, and 4 . The important results apparent in the figure are summarized as follows:

(1) The ASE increases as the number of the next-hop nodes increases.

(2) The ASE increases as $\gamma^{S \mathrm{NH}}$ increases.

(3) The ASE is 0.5 for DF even when $\gamma^{S N H}$ increases.

Figure 4 shows the comparison of average spectral efficiency using JNRS protocol and DF for $L=1,2$ and $M=1,2$.
The important results apparent in the figure are summarized as follows:

(1) The ASE reduces as number of relay nodes increases.

(2) The ASE increases as $\gamma^{\mathrm{SNH}}$ increases.

(3) The ASE increases as number of the next-hop nodes increases.

(4) The ASE is 0.5 for DF even when $\gamma^{\text {SNH }}$ increases.

Figure 5 shows the comparison of outage probability useing JNRS protocol and DF for $L=1,2$ and $M=1,2$. The important results apparent in the figure are that the outage probability reduces as the number of relay and next-hop nodes increases. The outage probability is higher for DF compared to JNRS.

\section{Conclusion}

We presented a JNRS protocol for a DF cooperative network. The proposed protocol has been shown to be effective in providing spectral efficiency and diversity gain. We illustrated the benefit of joint next-hop and relay nodes selection in 


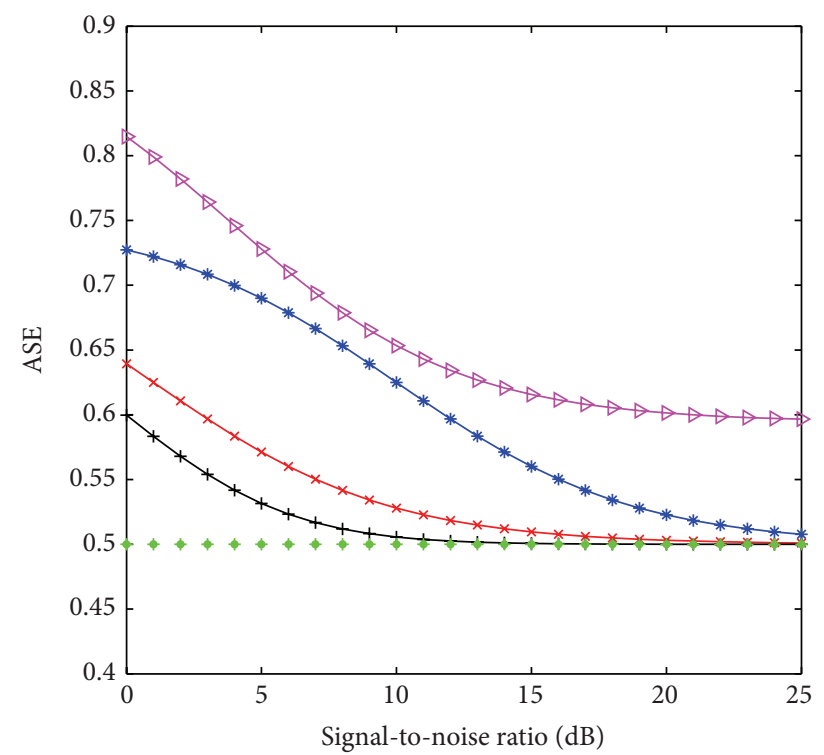

$$
\begin{aligned}
& \longrightarrow \text { DF-JNRS, } L=1, M=1, \gamma^{S N H}=1 \mathrm{~dB} \\
& \because \text { DF-JNRS, } L=1, M=2, \gamma^{S N H}=1 \mathrm{~dB} \\
& \longrightarrow \text { DF-JNRS, } L=1, M=1, \gamma^{S N H}=10 \mathrm{~dB} \\
& \rightarrow \text { DF-JNRS, } L=2, M=1, \gamma^{S N H}=2 \mathrm{~dB} \\
& \longrightarrow \text { DF, } M=1
\end{aligned}
$$

Figure 4: The ASE for $L=1,2$ and $M=1,2$ and different signalto-noise ratio.

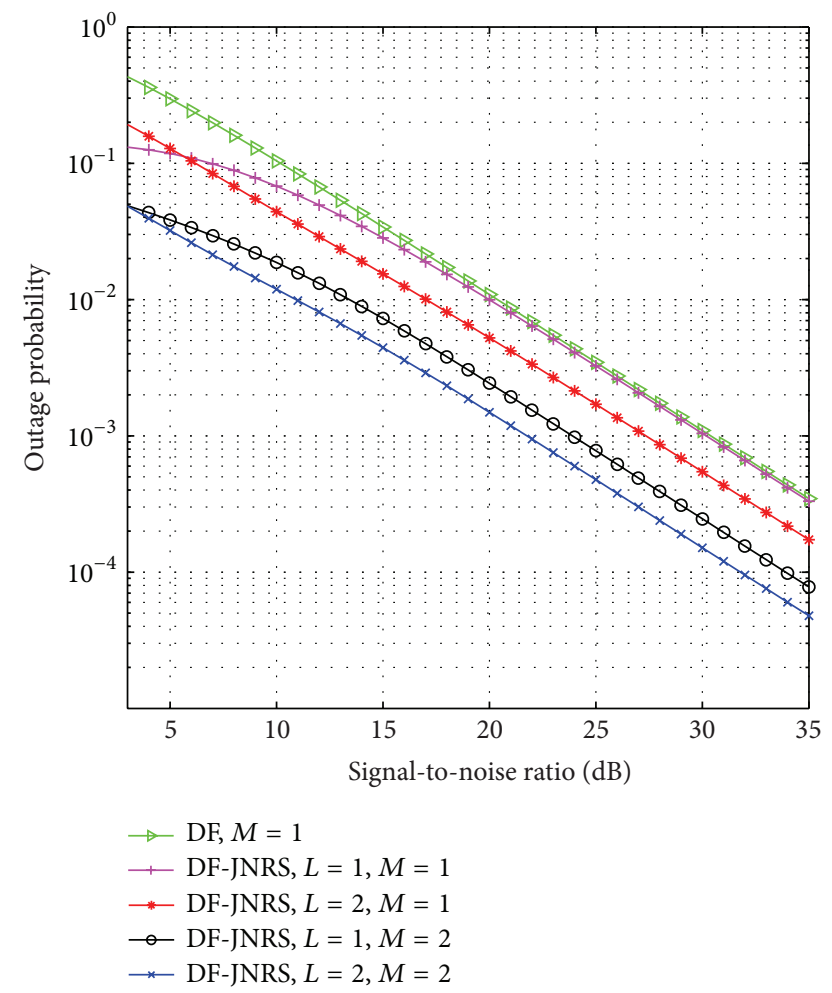

FIgURE 5: Outage probability for $L=1,2$ and $M=1,2$, and $\beta=1$ and different signal-to-noise ratio. providing less spectral efficiency loss and outage probability compared to DF protocol. We have observed that if the next-hop nodes increase, the spectral efficiency and outage probability loss reduce. In addition, if the number of relay nodes increased, the spectral efficiency loss increases and the outage probability reduces. As future work and, for the dense network, multiple next-hop and relay nodes can be selected in cooperation which can improve the system performance compared to selecting a single next-hop and relay nodes.

\section{Conflict of Interests}

The author declares that there is no conflict of interests regarding the publication of this paper.

\section{References}

[1] T. Rappaport, Wireless Communications: Principles and Practice, Prentice Hall, Upper Saddle River, NJ, USA, 2nd edition, 2002.

[2] L. Hanzo, O. Alamri, M. El-Hajjar, and N. Wu, Near-Capacity Multifunctional MIMO Systems, IEEE Press, John Wiley \& Sons, 2009.

[3] G. Kramer, M. Gastpar, and P. Gupta, "Cooperative strategies and capacity theorems for relay networks," IEEE Transactions on Information Theory, vol. 51, no. 9, pp. 3037-3063, 2005.

[4] A. Sendonaris, E. Erkip, and B. Aazhang, "User cooperation diversity. Part I. System description," IEEE Transactions on Communications, vol. 51, no. 11, pp. 1927-1938, 2003.

[5] A. Stefanov and E. Erkip, "Cooperative coding for wireless networks," IEEE Transactions on Communications, vol. 52, no. 9, pp. 1470-1476, 2004.

[6] T. Hunter and A. Nosratinia, "Diversity through coded cooperation," IEEE Transactions on Wireless Communications, vol. 5, no. 2, pp. 283-289, 2006.

[7] J. N. Laneman, D. N. C. Tse, and G. W. Wornell, "Cooperative diversity in wireless networks: efficient protocols and outage behavior," IEEE Transactions on Information Theory, vol. 50, no. 12, pp. 3062-3080, 2004.

[8] L. Zheng and D. N. C. Tse, "Diversity and multiplexing: a fundamental tradeoff in multiple-antenna channels," IEEE Transactions on Information Theory, vol. 49, no. 5, pp. 10731096, 2003

[9] A. Alkhayyat, O. Gazi, and S. B. Sadkhan, "The role of delay and connectivity in throughput reduction of cooperative decentralized wireless networks," Mathematical Problems in Engineering, vol. 2015, Article ID 294016, 10 pages, 2015.

[10] Y. Jing and B. Hassibi, "Distributed space-time coding in wireless relay networks," IEEE Transactions on Wireless Communications, vol. 5, no. 12, pp. 3524-3536, 2006.

[11] Y. Liu, X.-G. Xia, and H. Zhang, "Distributed space-time coding for full-duplex asynchronous cooperative communications," IEEE Transactions on Wireless Communications, vol. 11, no. 7, pp. 2680-2688, 2012.

[12] H. Ochiai, P. Mitran, and V. Tarokh, "Variable-rate two-phase collaborative communication protocols for wireless networks," IEEE Transactions on Information Theory, vol. 52, no. 9, pp. 4299-4313, 2006.

[13] E. Beres and R. Adve, "Selection cooperation in multi-source cooperative networks," IEEE Transactions on Wireless Communications, vol. 7, no. 1, pp. 118-127, 2008. 
[14] A. Bletsas, A. Khisti, and M. Z. Win, "Opportunistic cooperative diversity with feedback and cheap radios," IEEE Transactions on Wireless Communications, vol. 7, no. 5, pp. 1823-1827, 2008.

[15] F. A. Onat, A. Adinoyi, Y. Fan, H. Yanikomeroglu, J. S. Thompson, and I. D. Marsland, "Threshold selection for SNR-based selective digital relaying in cooperative wireless networks," IEEE Transactions on Wireless Communications, vol. 7, no. 11, pp. 4226-4237, 2008.

[16] A. S. Ibrahim, A. K. Sadek, W. Su, and K. J. R. Liu, "Cooperative communications with relay-selection: when to cooperate and whom to cooperate with?" IEEE Transactions on Wireless Communications, vol. 7, no. 7, pp. 2814-2827, 2008.

[17] R. Hkmat, Ad-Hoc Networks: Fundamental Properties and Network Topologies, Springer, 2006.

[18] A. Goldsmith, Wireless Communication, Cambridge University Press, 2005.

[19] O. Simeone, I. Stanojev, S. Savazzi, Y. Bar-Ness, U. Spagnolini, and R. Pickholtz, "Spectrum leasing to cooperating secondary ad hoc networks," IEEE Journal on Selected Areas in Communications, vol. 26, no. 1, pp. 203-213, 2008.

[20] T. Koch, "Is the assumption of perfect channel-state information in fading channels a good assumption?" in Proceedings of the 2nd International Symposium on Applied Sciences in Biomedical and Communication Technologies (ISABEL '09), pp. 1-6, IEEE, Bratislava, Slovakia, November 2009.

[21] A. Bletsas, A. Khisti, D. P. Reed, and A. Lippman, "A simple cooperative diversity method based on network path selection," IEEE Journal on Selected Areas in Communications, vol. 24, no. 3, pp. 659-672, 2006. 


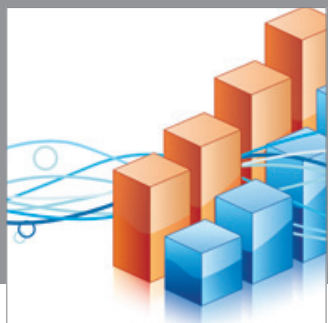

Advances in

Operations Research

mansans

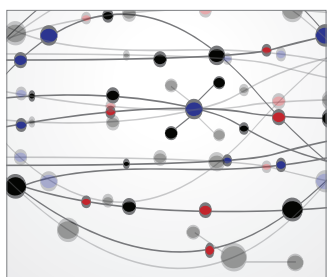

The Scientific World Journal
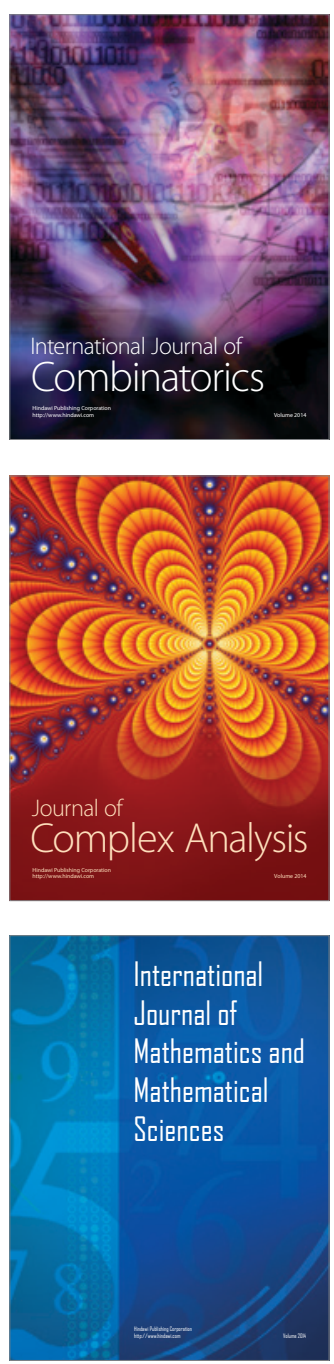
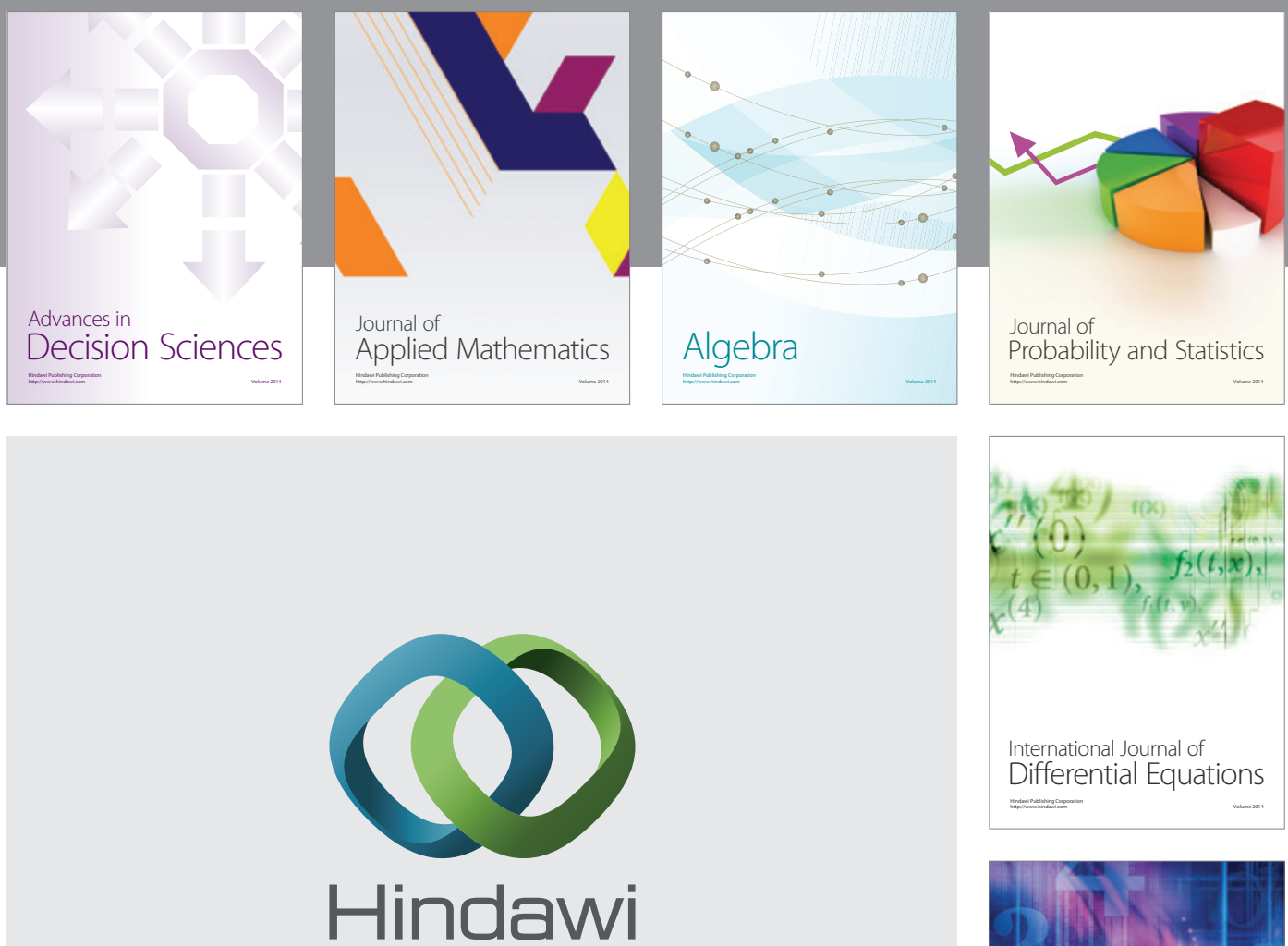

Submit your manuscripts at http://www.hindawi.com
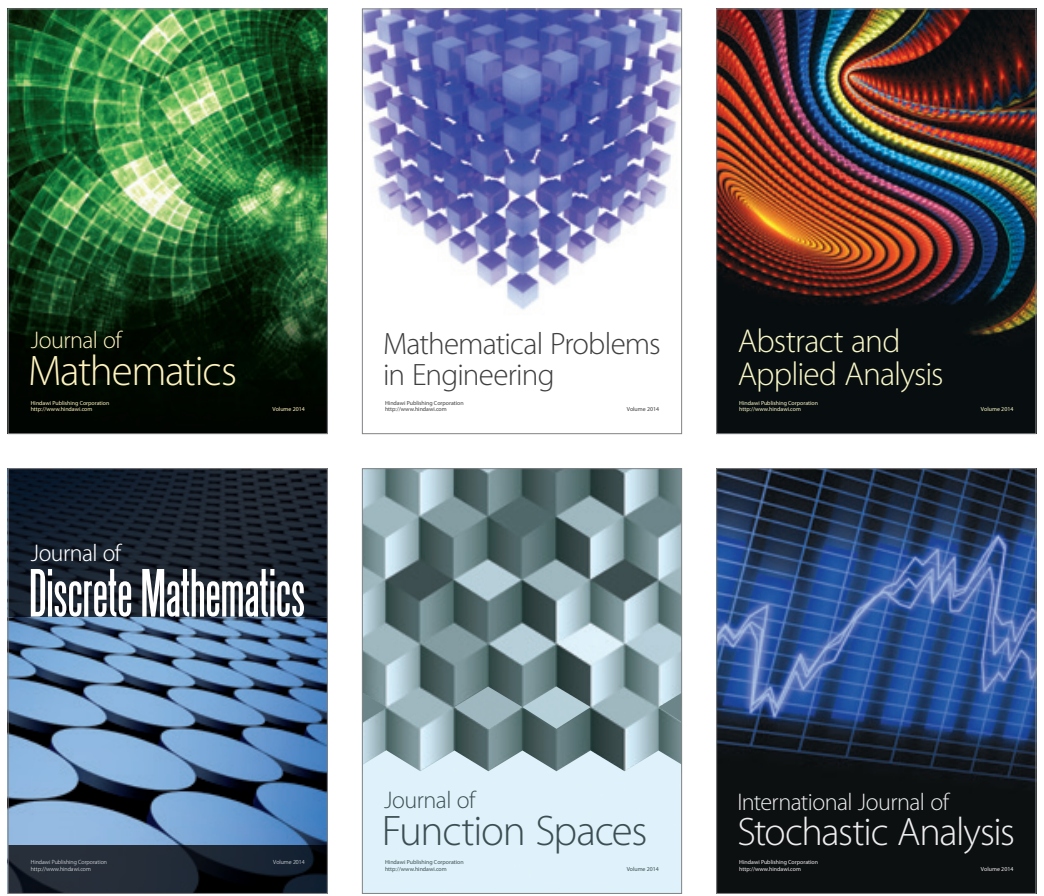

Journal of

Function Spaces

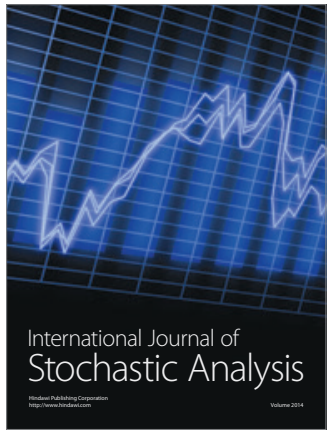

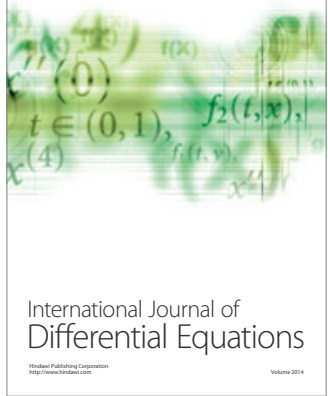
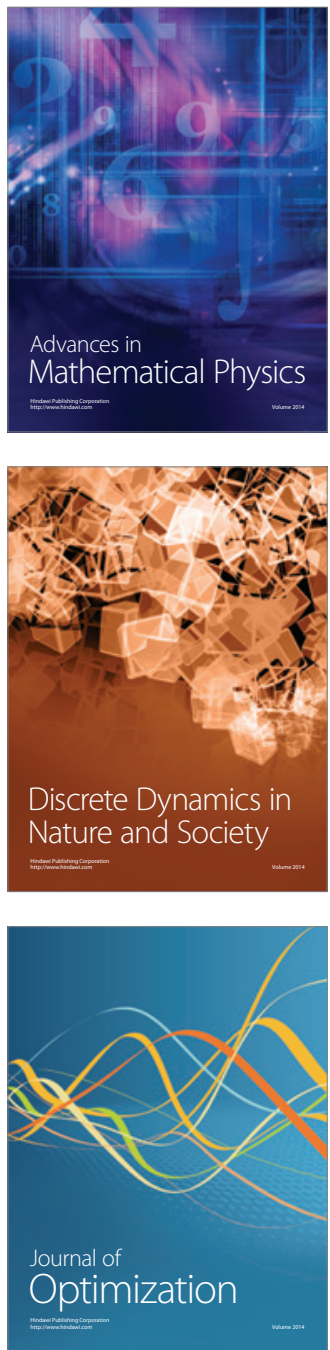